\title{
Sinking skin flap syndrome in a patient with bone resorption after cranioplasty and ventriculoperitoneal shunt placement: illustrative case
}

\author{
Camryn R. Rohringer, BSc, ${ }^{1}$ Taryn J. Rohringer, $M D,{ }^{2}$ Sumit Jhas, $M D,{ }^{3}$ and Mehdi Shahideh, $\mathrm{MD}^{3}$ \\ ${ }^{1}$ Physical Sciences, Sunnybrook Research Institute, Toronto, Ontario, Canada; ${ }^{2}$ Diagnostic Radiology, University of Toronto, Toronto, Ontario, Canada; and ${ }^{3}$ Neurosurgery, \\ Trillium Health Partners, Mississauga, Ontario, Canada
}

\begin{abstract}
BACKGROUND Sinking skin flap syndrome (SSFS) is an uncommon complication that can follow decompressive craniectomy. Even less common is the development of SSFS following bone resorption after cranioplasty with exacerbation by a ventriculoperitoneal (VP) shunt.

OBSERVATIONS A 56-year-old male sustained a severe traumatic brain injury and subsequently underwent an emergent decompressive craniectomy. After craniectomy, a cranioplasty was performed, and a VP shunt was placed. The patient returned to the emergency department 5 years later with leftsided hemiplegia and seizures. His clinical presentation was attributed to complete bone flap resorption (BFR) complicated by SSFS likely exacerbated by his VP shunt and the resultant mass effect on the underlying brain parenchyma. The patient underwent surgical intervention via synthetic bone flap replacement. Within 6 days, he recovered to his baseline neurological status.
\end{abstract}

LESSONS SSFS after complete BFR is a rare complication following cranioplasty. To the authors' knowledge, having a VP shunt in situ to exacerbate the clinical picture has yet to be reported in the literature. In addition to presenting the case, the authors also describe an effective treatment strategy of decompressing the brain and elevating the scalp flap while addressing the redundant tissue, then using a synthetic mesh to reconstruct the calvarial defect while keeping the shunt in situ.

https://thejns.org/doi/abs/10.3171/CASE21359

KEYWORDS sinking skin flap syndrome; craniectomy; craniotomy; cranioplasty; ventriculoperitoneal shunt; hydrocephalus

Decompressive craniectomies can be performed in medically refractory cases of increased intracranial pressure, including in the treatment of malignant edema from traumatic brain injury. Post-traumatic hydrocephalus is a complication that not uncommonly follows decompressive craniectomy, ${ }^{1}$ with incidence rates ranging from $11.9 \%$ to $36 \% .^{2}$ A prevalent method of treatment for hydrocephalus is the implantation of a ventriculoperitoneal (VP) shunt, which drains cerebrospinal fluid (CSF) from the ventricles into the peritoneum, thereby lowering intracranial pressure. ${ }^{3}$

Cranioplasty can be performed after a craniectomy for protective, cosmetic, and physiological reasons and comes with its own set of potential complications following the procedure. One common complication that arises when autologous bone flaps are used is bone flap resorption $(\mathrm{BFR}),{ }^{4}$ and a less common complication is that of sinking skin flap syndrome (SSFS). BFR leads to disintegration of the replaced bone flap, causing disfiguration and a lack of skull protection that often requires reimplantation of a bone flap. ${ }^{5}$ SSFS may also occur after decompressive craniectomy; however, in contrast to BFR, SSFS more commonly transpires before a bone flap is implanted. ${ }^{6}$ When atmospheric pressure exceeds intracranial pressure, the skin flap begins to depress the underlying brain tissue, ultimately leading to neurological deterioration. ${ }^{7}$ Such deterioration can produce symptoms of headache, dysautonomia, chronic fatigue, seizures, impaired vigilance, motor deficits, and visual symptoms. ${ }^{8-10}$

In the current case report and review, we discuss a patient who, after decompressive craniotomy following traumatic brain injury and subsequent VP shunt placement, presented with neurological deterioration. He had experienced complete BFR followed by SSFS.

ABBREVIATIONS BFR = bone flap resorption; CSF = cerebrospinal fluid; CT = computed tomography; GCS = Glasgow Coma Scale; ICU = intensive care unit; $\mathrm{MRI}=$ magnetic resonance imaging; SSFS = sinking skin flap syndrome; VP = ventriculoperitoneal.

INCLUDE WHEN CITING Published September 13, 2021; DOI: 10.3171/CASE21359

SUBMITTED June 15, 2021. ACCEPTED August 5, 2021.

(C) 2021 The authors, CC BY-NC-ND 4.0 (http://creativecommons.org/licenses/by-nc-nd/4.0/). 
Though cases of sunken flaps after decompressive craniectomy have been reported in the literature, this case is unique in that it followed cranioplasty and complete BFR, with a VP shunt being in place to exacerbate the sunken flap.

\section{Illustrative Case}

A 56-year-old man presented to the emergency department after a traumatic fall. His past medical history was remarkable only for atrial fibrillation, and he was not receiving anticoagulants. On presentation, his Glasgow Coma Scale (GCS) score was 13, and computed tomography (CT) of the head revealed a 4-cm right temporal hematoma with acute subdural and subarachnoid blood, a left subdural hematoma, and skull fractures (Fig. $1 A$ and $B$ ). At the time of neurosurgical assessment, his condition had deteriorated to a GCS score of 11 , he was hemiplegic on the right side, and his Injury Severity Score was critical at 25. A second CT was performed to assess the evolution of his injuries causing this clinical deterioration, which demonstrated enlargement of the hematoma to $5 \mathrm{~cm}$, now with substantial mass effect causing a midline shift. A right decompressive hemicraniotomy and hematoma evacuation was performed (Fig. 1C and D). After surgery, he was admitted to the intensive care unit (ICU) for monitoring.

While the patient was convalescing in the ICU, his level of consciousness began deteriorating at approximately 1 month after craniectomy. CT of the head revealed hydrocephalus, and the patient was taken for a bone flap replacement, or cranioplasty, to resolve this issue. Two weeks after this procedure, the patient did not demonstrate improvement clinically or radiographically; consequently, a
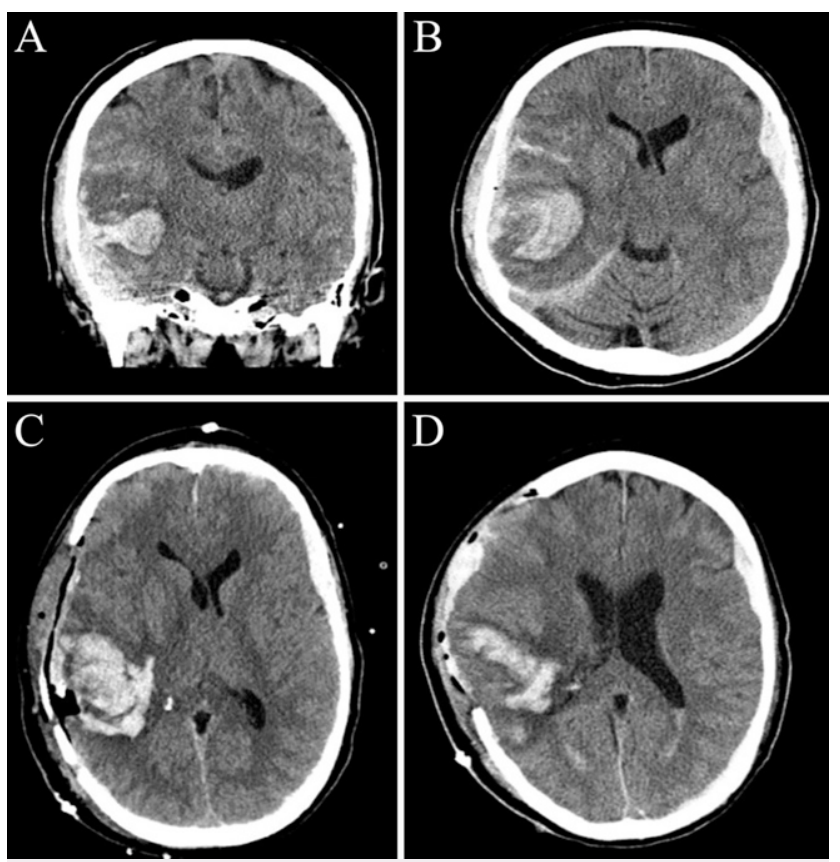

FIG. 1. CT of the head. A: Preoperative axial cross-section showing 4-cm right temporal hematoma with acute subdural, subarachnoid blood and a left subdural hematoma with associated midline shift. B: Preoperative coronal cross-section. C: Axial cross-section on postoperative day 1. D: Axial cross-section on postoperative day 5 showing the evolution of the hematoma and outward herniation of the brain through craniectomy defect. left VP shunt was placed to resolve his hydrocephalus. He demonstrated clinical improvement postoperatively and eventually was downgraded to a ward bed. Within 1 month's time, he was deemed safe for discharge to an inpatient rehabilitation center. He spent a total of 3 months in an acute care facility.

The patient was seen at 3 months and 5 months after discharge. He was demonstrating neurological improvement from his original clinical presentation. Aside from some cognitive deficits, he had a full motor recovery. His incision and bone flap were healing well. The patient was then lost to follow-up and was next seen nearly 3 years after these visits. He returned for medical attention after visiting the emergency department with a cranial defect at the craniectomy site. He denied headaches, weakness, or any other neurological deficits. A CT scan and radiograph demonstrated BFR at the posterior aspect of the flap with a cavity noted (Fig. 2). The rest of the flap had healed. The bony edges were soft and did not appear to be lacerating the skin flap. He was reconnected with the neurosurgical team by the emergency physician. At his clinic visit, management options were discussed, including the possibility of leaving the hole as it currently existed because it posed no significant safety concerns or placing a synthetic bone flap for mainly cosmetic reasons. The patient did not attend follow-up after this conversation, and we were unable to contact him.

The patient returned to his neurologist 5 years after his initial presentation to the emergency department, complaining of mild leftsided weakness and tremors. CT and magnetic resonance imaging (MRI) were ordered (Fig. 3). Immediately after undergoing MRI, the patient presented to the emergency department with left-sided hemiplegia and left upper extremity focal seizures, which manifested in the form of tremors. MRI of the brain revealed vasogenic edema in the compressed brain surrounding an enlarged, sunken craniectomy cavity involving the right posterior, frontal, and parietal lobes. The cavity measured almost $8 \mathrm{~cm}$ in its largest diameter with a depth of $4 \mathrm{~cm}$ (Fig. 3B). Complete bone resorption led to the development of SSFS. A considerable amount of time was first spent cleaning out the cavity, which housed years' worth of hair, desquamated skin, and slimy residue likely from hair care products (Fig. 4A and B). Because the patient was unable to see the defect, given its location, it simply was never cleaned, and it had accumulated a significant amount of debris. After thorough extracranial cleaning with peroxide, betadine, and chlorhexidine, the patient was taken to the operating room emergently for elevation of the sunken
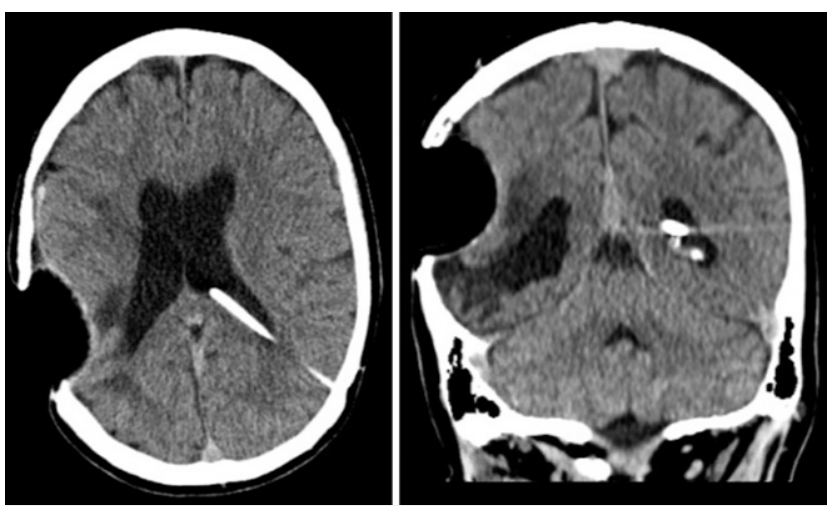

FIG. 2. CT of the head. Left: Axial view of a cavity measuring $6 \mathrm{~cm}$ in diameter at the posterior aspect of the flap. Right: Coronal view of the same cavity. Lack of both edema and midline shift is noted. 

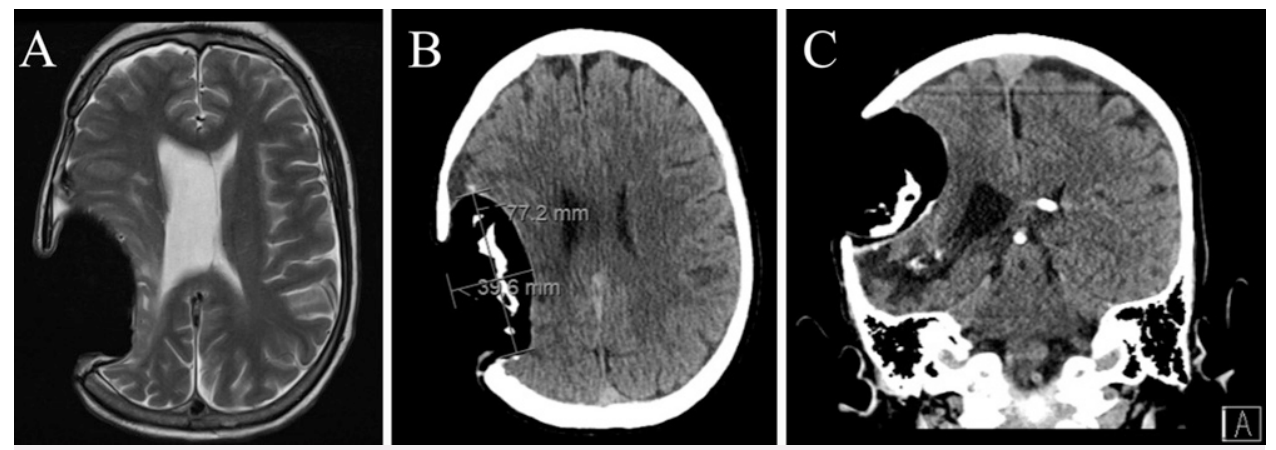

FIG. 3. A: MRI of the brain. T2-weighted axial cross-section in August 2020 showing progression of the cavity size with evidence of cortical edema from the mass effect. Image was obtained just before the patient presented to the emergency department. B: CT of the head. Axial cross-section showing sunken cavity measuring $7.7 \mathrm{~cm}$ in length with a depth of $4 \mathrm{~cm}$ at the posterior aspect of the flap. C: CT of the head. Coronal cross-section showing the same cavity with distortion of the underlying cortex. Buildup of debris is noted within the cavity.

flap and a synthetic cranioplasty. No superficial wound swabs were sent for culture, because the area had been debrided and cleaned with antibacterial solutions that would render culture yield low, and there was a low index of suspicion for an underlying infection originating at the skin and progressing intracranially. Elevating the skin flap tissue from the concave cavity was a challenge because some areas were extremely adherent to the underlying brain tissue. Once the skin was elevated off the brain, the dural defect was covered with a $4 \times 5$-in synthetic duraplasty. The bone edges were shaved using the pneumatic drill until an area was reached that demonstrated normal thickness and good health. A titanium synthetic mesh was fashioned and secured using 4-mm screws. The excess skin that comprised the cavity was carefully excised and reapproximated. The scalp flap was then reapproximated and closed. The removed bone flap was sent for culture analysis along with swabs of the epidural space, yielding no sign of growth. Postoperatively, the patient's incision remained clean and dry, with a small amount of yellow discharge at the posterior aspect of the incision, for which he was administered cefazolin (Fig. 4C). On postoperative day 6, the patient was back to his baseline status and cleared for discharge to an inpatient rehabilitation center, as determined necessary by physiotherapy and occupational therapy. Appropriate followup with neurology and neurosurgery was arranged.
Three months after discharge, the patient was seen in virtual follow-up and demonstrated neurological recovery, with resolution of his hemiplegia and no further episodes of seizure. He denied any headache, change in vision, or weakness. He showed no signs of infection at the incision site, and there were no concerning findings on MRI and CT (Fig. 5).

\section{Discussion \\ Observations}

There have been several cases of SSFS with subsequent neurological deterioration reported in the literature since Yamamura first proposed the concept in 1977. 7,11,12 Typical cases begin with patients undergoing a decompressive craniectomy for brain injury, stroke, or postoperative complications, with no replacement of the bone flap, then later presenting with symptoms of cortical dysfunction, including dizziness, headache, changes in mood, or seizures. ${ }^{7,11}$ Upon examination of these patients, a depressed skin flap is revealed.

When a bone flap is replaced, reabsorption tends to involve the entire flap. In this case, the reabsorption was atypical, commencing in the posterior aspect of the flap. Nothing notable in this case could be discerned that may have predisposed the posterior aspect of the flap to such reabsorption; however, Kim et al. noted in their review on bone flap resorption after cranioplasty that, with multiple
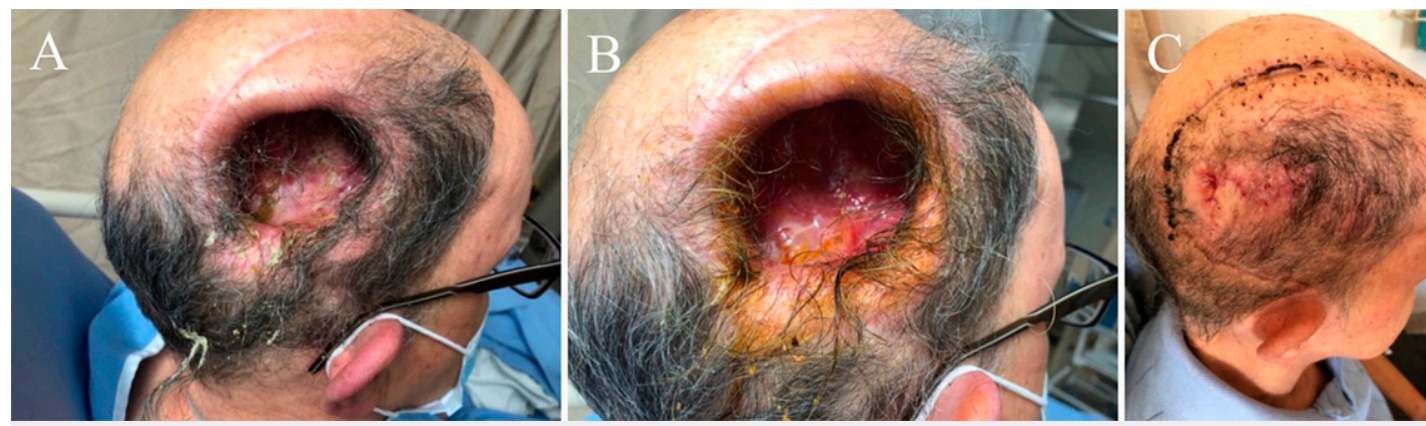

FIG. 4. A: Photo of the cranial defect taken in the emergency department before cleaning of cavity. B: Photo of the cranial defect taken in the emergency department after rudimentary cleaning of cavity. C: Photo taken at 6-week follow-up after cranioplasty. Incision along the craniotomy site is healing nicely. The incision required to excise the excess tissue within the cavity has completely healed with no evidence of redundant tissue. 

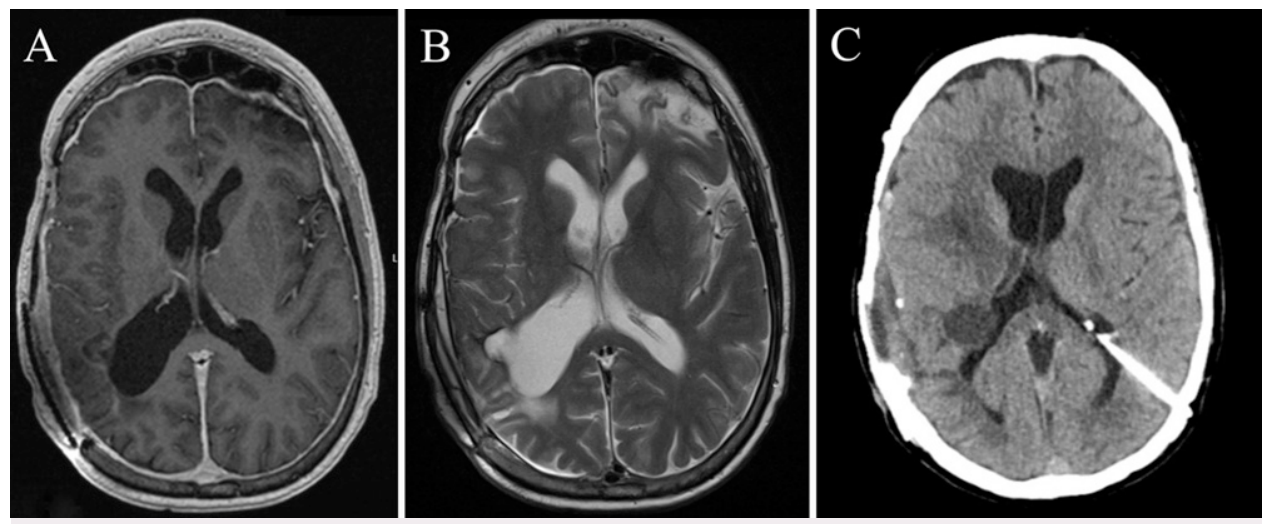

FIG. 5. A: MRI of the brain. Axial cross-section with gadolinium enhancement at 3 months postoperatively. B: MRI of the brain. Axial T2-weighted cross-section at 3 months postoperatively shows resolution of the right parietal edema and mass effect. C: CT of the head. Axial cross-section at 3-month follow-up. Titanium cranioplasty with a small residual subdural collection is noted. Complete resolution of mass effect and midline shift.

fractures, a larger number of bony fragments are more likely to denature bone proteins and impede osteogenesis. ${ }^{13}$ It is possible that a separate fracture fragment was near the posterior section of the flap, thus contributing to the development of a focal rather than the typically diffuse process of bone reabsorption. Exact mechanisms contributing to bone reabsorption are still not well understood, ${ }^{14}$ and no definite conclusion can be made regarding why the reabsorption was localized to one area, but this represents a viable potential explanation.

Regarding the pathophysiology of the sunken flap, several ideas have been theorized. Older explanations include that it is the pulsating movements of arterial or venous pressure intracranially that are responsible for the sunken flap. ${ }^{12}$ Another theory discusses how atmospheric pressure is transmitted to the intracranial cavity, forcing the scalp to inwardly involute over the cranial defect, leading to paradoxical herniation and a sunken skin flap that causes neurological dysfunction. ${ }^{15}$ Others mention the possibility of the skull defect generating a siphon effect on CSF dynamics involving a distortion of the dura, cortex, and venous return through scarring and direct pressure. $^{16-18}$ Last, and most relevant to the case presented here, neurological deterioration has also been theorized to arise from a negative gradient between atmospheric and intracranial pressure that is exacerbated by CSF compartment changes after CSF hypovolemia. ${ }^{19,20}$ This hypovolemia can arise from the insertion of a VP shunt, which is often inserted in craniectomy patients to relieve hydrocephalus.

In this case, the last theory encompasses what likely took place. Due to the development of hydrocephalus, a VP shunt was inserted into this patient to resolve the issue. The most similar case identified occurred in 2008, when a patient who underwent a decompressive craniectomy developed hydrocephalus and had a VP shunt inserted. The next day, the skin flap had severely depressed, and the patient was experiencing symptoms of neurological dysfunction. After the valve pressure was increased and a cranioplasty was performed for the sunken flap, the patient successfully recovered. ${ }^{21}$ If we had tied off the shunt, it is possible that this patient would have experienced improvement similar to that in the 2008 case; however, because of the combination of, first, the need for a definitive, time-conscious management strategy due to the patient's significant neurological deficits, and, second, a history of this patient being lost to follow-up, we thought in the current case that halting shunt activity was not the preferred treatment strategy, because it would require regular followup. Further contrast between the 2008 case and the one detailed here is that although this case's patient underwent a craniectomy, he already had undergone a cranioplasty with bone flap replacement, so there was no immediate concern that the pressure gradient between atmospheric pressure and CSF pressure would cause SSFS, because there was bone in place to prevent this phenomenon. However, because this patient was simultaneously experiencing BFR, the CSF compartment pressure changes from shunting in conjunction with the BFR brought the intracranial pressure to below atmospheric level, generating a sunken flap and the patient's presentation of hemiplegia and seizures. This unique constellation of post-craniectomy findings had yet to be reported in the literature.

In light of the debris found in the patient's head cavity, there was concern for infection acting as the underlying predisposing factor to subsequent osteolysis. Specifically, the presence of and subsequent low-grade infection caused by Propionibacterium acnes has been labeled a risk factor for BFR. ${ }^{22,23}$ Although the etiology of this infection is poorly understood, ${ }^{24}$ it is realized that low-grade infection takes place on the bone flap itself rather than on the skin above the flap. ${ }^{22,24}$ Thus, in our case, infection was not of great concern, because the debris was in the extracranial cavity and not communicating intracranially. Intraoperatively, as mentioned in the illustrative case, the resected bone flap was sent for culture analysis, which found no growth.

Regarding the key role of the VP shunt in this case in contributing to the negative pressure gradient, one recent article surveyed experiences and lessons learned regarding the timing of performing a craniotomy when a VP shunt is present. The authors mention that although the presence of a VP shunt is a known risk factor for complications in cranioplasty, there is minimal literature on optimal timing of VP shunt insertion and cranioplasty when patients are receiving both procedures. Some reports mention a higher risk of overall complications and bone resorption if performed simultaneously, whereas others pose the opposite. ${ }^{25-28}$

To explore optimal timing for shunt placement in relation to cranioplasty, one 2017 study by Meyer et al. ${ }^{26}$ retrospectively analyzed outcomes of patients who underwent cranioplasty and VP shunt placement. The subgroup of patients who had these procedures 
simultaneously performed compared with those who underwent the procedures separately revealed non-statistically significant differences in outcomes of infection and mechanical failure. ${ }^{26}$ Contrastingly, a 2015 study compared the same subgroup categories and found the simultaneous timing group to have a complication rate of $47 \%$ versus $12 \%$ in the group that had staged cranioplasty and VP shunt procedures. ${ }^{28}$ These two examples illustrate the lack of consensus that currently exists in the literature with respect to the safety of performing these procedures in close proximity to each other.

\section{Lessons}

This report illustrates a unique case of superimposition of multiple complications after traumatic head injury and decompressive craniotomy. The risk of sunken flap syndrome and resultant devastating neurological outcomes was highlighted, particularly in a case after cranioplasty with BFR and a VP shunt exacerbating SSFS. It additionally demonstrates the management strategy undertaken as an effective one: decompressing the brain and flap and using a synthetic construct to reconstruct the calvarial defect while keeping the shunt in situ.

\section{References}

1. Wang QP, Ma JP, Zhou ZM, You C. Impact of operation details on hydrocephalus after decompressive craniectomy. Neurosciences (Riyadh). 2016;21(1):10-16.

2. Vedantam A, Yamal JM, Hwang H, Robertson CS, Gopinath SP. Factors associated with shunt-dependent hydrocephalus after decompressive craniectomy for traumatic brain injury. J Neurosurg. 2018;128(5):1547-1552.

3. Gholampour S, Bahmani M, Shariati A. Comparing the efficiency of two treatment methods of hydrocephalus: shunt implantation and endoscopic third ventriculostomy. Basic Clin Neurosci. 2019;10(3): 185-198.

4. Korhonen TK, Salokorpi N, Ohtonen P, et al. Classification of bone flap resorption after cranioplasty: a proposal for a computed tomography-based scoring system. Acta Neurochir (Wien). 2019;161(3): 473-481.

5. Schütz A, Murek M, Stieglitz LH, et al. ACE-inhibitors: a preventive measure for bone flap resorption after autologous cranioplasty? J Neurosurg. 2019;131:1607-1614.

6. Hakmi H, Joseph DK, Sohail A, Tessler L, Baltazar G, Stright A. Sinking skin flap syndrome in the multi-trauma patient: a paradoxical management to TBI post craniectomy. J Surg Case Rep. 2020;2020:rjaa172.

7. Khan NAJ, Ullah S, Alkilani W, Zeb H, Tahir H, Suri J. Sinking skin flap syndrome: phenomenon of neurological deterioration after decompressive craniectomy. Case Rep Med. 2018;2018:9805395.

8. Boccagni C, Bagnato S, Alaimo V, Galardi G. Neurologic deterioration in sinking skin flap syndrome after diuretic therapy. Neurol Clin Pract. 2021;11(4):e561-e563.

9. Chugh A, Punia P, Gotecha S. Sinking skin flap syndrome following posttraumatic hydrocephalus. Case Rep Neurol Med. 2021;2021: 6682310.

10. Fawley N, Udeh C. Sinking skin flap syndrome after decompressive craniectomy: a case report. A A Pract. 2018;11(9):241-243.

11. Cho H, Kim CH, Kim JH, Kim JM. Paradoxical herniation after decompressive craniectomy for acute subdural hematoma. J Korean Neurosurg Soc. 2006;40(1):51-53.

12. Schiffer J, Gur R, Nisim U, Pollak L. Symptomatic patients after craniectomy. Surg Neurol. 1997;47(3):231-237.

13. Kim JS, Cheong JH, Ryu JI, Kim JM, Kim CH. Bone flap resorption following cranioplasty after decompressive craniectomy: preliminary report. Korean J Neurotrauma. 2015;11(1):1-5.
14. de França SA, Nepomuceno TB, Paiva WS, Andrade AF, Teixeira MJ, Tavares WM. Cranial autologous bone flap resorption after a cranioplasty: a case report. Surg Neurol Int. 2018;9:61.

15. Langfitt TW. Increased intracranial pressure. Clin Neurosurg. 1969;16:436-471.

16. Fodstad H, Love JA, Ekstedt J, Fridén H, Liliequist B. Effect of cranioplasty on cerebrospinal fluid hydrodynamics in patients with the syndrome of the trephined. Acta Neurochir (Wien). 1984;70(1-2): 21-30.

17. Richaud J, Boetto S, Guell A, Lazorthes Y. Effects of cranioplasty on neurological function and cerebral blood flow. Article in French. Neurochirurgie. 1985;31(3):183-188.

18. Segal DH, Oppenheim JS, Murovic JA. Neurological recovery after cranioplasty. Neurosurgery. 1994;34(4):729-731.

19. Oyelese AA, Steinberg GK, Huhn SL, Wijman CA. Paradoxical cerebral herniation secondary to lumbar puncture after decompressive craniectomy for a large space-occupying hemispheric stroke: case report. Neurosurgery. 2005;57(3):E594.

20. Schwab S, Erbguth F, Aschoff A, Orberk E, Spranger M, Hacke W. ["Paradoxical" herniation after decompressive trephining. Article in German. Nervenarzt. 1998;69(10):896-900.

21. Han PY, Kim JH, Kang HI, Kim JS. "Syndrome of the sinking skinflap" secondary to the ventriculoperitoneal shunt after craniectomy. J Korean Neurosurg Soc. 2008;43(1):51-53.

22. Butenschoen VM, Seifert M, Meyer B, Krieg SM. Presence of Propionibacterium acnes in patients with aseptic bone graft resorption after cranioplasty: preliminary evidence for low-grade infection. J Neurosurg. 2020;133(3):912-917.

23. Göttsche J, Mende KC, Schram A, et al. Cranial bone flap resorption-pathological features and their implications for clinical treatment. Neurosurg Rev. 2021;44(4):2253-2260.

24. Shlobin NA, Palmer AH, Kam KL, Brat DJ, Potts MB. Bone flap resorption associated with indolent Propionibacterium acnes infection after cranioplasty: case report with pathological analysis. World Neurosurg. 2020;138:313-316.

25. Heo J, Park SQ, Cho SJ, Chang JC, Park HK. Evaluation of simultaneous cranioplasty and ventriculoperitoneal shunt procedures. J Neurosurg. 2014;121(2):313-318.

26. Meyer RM 4th, Morton RP, Abecassis IJ, et al. Risk of complications with simultaneous cranioplasty and placement of ventriculoperitoneal shunt. World Neurosurg. 2017;107:830-833.

27. Mustroph CM, Malcolm JG, Rindler RS, et al. Cranioplasty infection and resorption are associated with the presence of a ventriculoperitoneal shunt: a systematic review and meta-analysis. World Neurosurg. 2017;103:686-693.

28. Schuss P, Borger V, Güresir Á, Vatter H, Güresir E. Cranioplasty and ventriculoperitoneal shunt placement after decompressive craniectomy: staged surgery is associated with fewer postoperative complications. World Neurosurg. 2015;84(4):1051-1054.

\section{Disclosures}

The authors report no conflict of interest concerning the materials or methods used in this study or the findings specified in this paper.

\section{Author Contributions}

Conception and design: Taryn J. Rohringer, Shahideh, Jhas. Acquisition of data: Taryn J. Rohringer, Shahideh. Drafting the article: Camryn R. Rohringer. Critically revising the article: Camryn R. Rohringer, Taryn J, Rohringer, Shahideh. Reviewed submitted version of manuscript: Camryn R. Rohringer, Taryn J. Rohringer.

\section{Correspondence}

Camryn R. Rohringer, Sunnybrook Research Institute, Toronto, ON, Canada. camryn.rohringer@sri.utoronto.ca 